\title{
Quasi-Normal Modes of Massless Scalar Field around the 5D Ricci-flat Black String
}

\author{
Molin Liu * Hongya Liu $\dot{\dagger}$ and Yuanxing Gu斻 \\ School of Physics and Optoelectronic Technology, \\ Dalian University of Technology, Dalian, 116024, P. R. China
}

\begin{abstract}
As one candidate of the higher dimensional black holes, the 5D Ricci-flat black string is considered in this paper. By means of a non-trivial potential $V_{n}$, the quasi-normal modes of a massless scalar field around this black string space is studied. By using the classical third order WKB approximation, we analyse carefully the evolution of frequencies in two aspects, one is the induced cosmological constant $\Lambda$ and the other is the quantum number $n$. The massless scalar field decays more slowly because of the existences of the fifth dimension and the induced cosmological constant. If extra dimension has in fact existed near black hole, those quasi-normal frequencies may have some indication on it.
\end{abstract}

PACS numbers: 04.70.Dy, 04.50.+h

Keywords: quasi-normal models; fifth dimension; black string.

\section{INTRODUCTION}

Through an additional field or perturbing the metric itself, the black hole suffers a damping oscillation phase. Usually, people call it Quasi-normal models (QNMs) or quasi-normal ringing. So, as the emission of gravitational wave $(\mathrm{GW})$, a normal model oscillation is replaced by the complex frequencies "quasi-normal" where the real part represents the actual frequency and the imaginary part represents the damping of the oscillation. Those frequencies are directly connected to the black hole's mass, charge, momentum and so on. In the view of QNMs' evolution, there are three stages: the first one is the initial outburst from the source of perturbation, the second one is the damping (quasi-normal) oscillation and the last one is the asymptotic tails at very late time. The evolution significantly depends on the asymptotic behavior of the space. In perturbation theory, the linear perturbation of static black holes was first studied by Regge and Wheeler in 1957 [1]. Soon after that, Vishveshwara [2] presented the QNMs by calculating the scattering of gravitational waves (GW) around a Schwarzschild black hole. Then Press [3] gave the original term

quasi-normal (QN) frequencies. This perturbations have been studied extensively in many literatures [4]. For the in-depth reviews, one can refer to [5] 6]. People believe that the QN frequencies could be detected by GW observatories (LIGO, VIRGO, TAMT, GEO600, and so on) in the future.

On the other hand, one kind of higher dimensional theory named induced matter theory is shown by Wesson and co-workers [7] 8] in the 90's of the last century. They showed a non-compact fifth dimension and pointed out the 4D source is induced from an empty 5D manifold. That is, 5D manifold is Ricci-flat while 4D hypersurface is curved by

\footnotetext{
${ }^{*}$ Electronic address: mlliudl@student.dlut.edu.cn

${ }^{\dagger}$ Electronic address: hyliu@dlut.edu.cn

${ }^{\ddagger}$ Electronic address: guiyx@dlut.edu.cn
} 
the 4D matters. So this theory is also called Space-Time-Matter (STM) theory. Meanwhile, in the STM framework, there are many extensive literatures discussing Quantum Dirac Equation [9], Perihelion Problem [10], Kaluza-Klein Solitons [11] [12], Black Hole [13] [14] [15] 16], Solar System Tests 18] and so on.

In the last decade, other robust extra dimensional models have appeared in gravitational-field theory such as ADD model [19] and Randall-Sundrum I model [20]/ II model [21] with the additional spacelike dimensions. In those models, our world is a 3-brane which is embedded in the higher dimensional space (bulk). To avoid interactions beyond any acceptable phenomenological limits, standard model (SM) particles (such as fermions, gauge bosons, Higgs) are confined on a $(3+1)$ dimensional hypersurface (3-brane) without accessing the transverse dimensions, except for the gravitons and scalar particles without charges under SM gauge group. In this paper, we assume the scalar field can freely propagate in the bulk.

If matter trapped on the brane undergoes gravitational collapse, a black hole will form naturally and its horizon extends into the extra dimension transverse to brane. Such higher dimensional object looked like a black hole on the brane is actually a black string in the higher dimensional brane world $[22]$. One natural candidate is a Schwarzschildde Sitter (SdS) black hole embedded into the 5D Ricci-flat space [13] [15] [16] [17]. It should be noticed that the STM theory is equivalent to the brane world [23] [24] [25].

Meanwhile, one of exciting predictions in large extra dimensional model [19] is that the CERN Large Hadron Collider (LHC) will produce black holes by the colliding of highly energetic particles when the scale of quantum gravity is near $\mathrm{TeV}$ [26]. Naturally, the detectable QNMs are studied widely in higher dimensional background [27]. In this paper, we calculate the QN frequency of massless scalar field around a 5D Ricci-flat black string space.

This paper is organized as follows: In Section II, the 5D Ricci-flat black string metric and the time-dependent radial equation about $R_{\omega}(r, t)$ are represented. In section III, by a tortoise coordinate transformation, the propagating master equation of scalar field is obtained. In section VI, by using the third order WKB method, the QN frequencies is obtained in Table I, II and III. Section V is a conclusion. We adopt the signature $(+,-,-,-,-)$ and put $\hbar, c$,and $G$ equal to unity. Lowercase Greek indices $\mu, \nu, \ldots$ will be taken to run over $0,1,2,3$ as usual, while capital indices $\mathrm{A}, \mathrm{B}, \mathrm{C}, \ldots$ run over all five coordinates $(0,1,2,3,4)$.

\section{KLEIN-GORDON EQUATION IN THE 5D RICCI-FLAT BLACK STRING SPACE}

A class of 5D black holes solutions have been presented by Mashhoon, Wesson and Liu [13] [15] [7] under STM scenario. Briefly, the static, three-dimensional spherically symmetric line element takes the form

$$
d S^{2}=\frac{\Lambda \xi^{2}}{3}\left[f(r) d t^{2}-\frac{1}{f(r)} d r^{2}-r^{2}\left(d \theta^{2}+\sin ^{2} \theta d \phi^{2}\right)\right]-d \xi^{2} .
$$

where $\xi$ is the open non-compact extra dimension coordinate. The part of this metric inside the square bracket is exactly the same line-element as the 4D Schwarzschild-de Sitter solution, which is bounded by two horizons - an inner horizon (black hole horizon) and an outer horizon (one may call this cosmological horizon).

The radial-dependent metric function $f(r)$ takes the form

$$
f(r)=1-\frac{2 M}{r}-\frac{\Lambda}{3} r^{2}
$$

where $\Lambda$ is the induced cosmological constant and $M$ is the central mass. The metric (1D) satisfies the $5 \mathrm{D}$ vacuum equation $R_{A B}=0$. Therefore, there is no cosmological constant when viewed from $5 \mathrm{D}$. But when viewed from $4 \mathrm{D}$, 
there is an effective cosmological constant $\Lambda$. So one can treat this $\Lambda$ as a parameter which comes from the fifth dimension. This solution has been studied in many works [28] [29] 30] [31] focusing mainly on the induced constant $\Lambda$, the extra force and so on.

We redefine the fifth dimension $\xi=\sqrt{3 / \Lambda} e^{\sqrt{\frac{\Lambda}{3}}} y$. With this redefinition, the metric (1) can be rewritten as

$$
d S^{2}=e^{2 \sqrt{\frac{\Lambda}{3}} y}\left[f(r) d t^{2}-\frac{1}{f(r)} d r^{2}-r^{2}\left(d \theta^{2}+\sin ^{2} \theta d \phi^{2}\right)-d y^{2}\right],
$$

Using the line element (11), the metric function (2) and above new extra dimension, a Randall-Sundrum (RS) type brane model is built up. Now, let us show the configuration in detail. There are two branes in this model: one brane is at $y=0$ and the other brane is at $y=y_{1}$. So the fifth dimension becomes finite. It could be very large as RS II model [20] or very small as RS I model [21]. The 4D line-element represents exactly the Schwarzschild-de Sitter black hole on a hypersurface ( $\xi$ or $y=$ constant). However, viewing from the $5 \mathrm{D}$ space, the horizon does not form a $4 \mathrm{D}$ sphere — it looks like a black string lying along the extra dimension. So, we call the solution (1) a 5D Ricci-flat black string solution.

The metric function (2) can be expressed by the horizons as follows

$$
f(r)=\frac{\Lambda}{3 r}\left(r-r_{e}\right)\left(r_{c}-r\right)\left(r-r_{o}\right) .
$$

The null hypersurface of this black string space is determined by its singularity $f(r)=0$. Obviously, the solutions to this equation are inner horizon $r_{e}$, outer horizon $r_{c}$ and a negative solution $r_{o}=-\left(r_{e}+r_{c}\right)$. The last one has no physical significance. Here we only consider the real solutions. $r_{c}$ and $r_{e}$ are given as

$$
\left\{\begin{array}{c}
r_{c}=\frac{2}{\sqrt{\Lambda}} \cos \eta, \\
r_{e}=\frac{2}{\sqrt{\Lambda}} \cos \left(120^{\circ}-\eta\right),
\end{array}\right.
$$

where $\eta=\frac{1}{3} \arccos (-3 M \sqrt{\Lambda})$ with $30^{\circ} \leq \eta \leq 60^{\circ}$. The real physical solutions are accepted only if $\Lambda$ satisfy $\Lambda M^{2} \leq \frac{1}{9}$ [13].

The massless scalar field $\Phi$ in the $5 \mathrm{D}$ black string space, satisfies the Klein-Gordon equation $\square \Phi=0$, where $\square=\frac{1}{\sqrt{g}} \frac{\partial}{\partial x^{A}}\left(\sqrt{g} g^{A B} \frac{\partial}{\partial x^{B}}\right)$ is the $5 \mathrm{D}$ d'Alembertian operator. We assume that the separable solutions are of the form

$$
\Phi=\frac{1}{\sqrt{4 \pi \omega}} \frac{1}{r} R_{\omega}(r, t) L(y) Y_{l m}(\theta, \phi)
$$

where $R_{\omega}(r, t)$ is the radial time-dependent function, $Y_{l m}(\theta, \phi)$ is the usual spherical harmonic function. The dependent equation about $R_{\omega}(r, t)$ in QNMs aspect is,

$$
-\frac{1}{f(r)} r^{2} \frac{\partial^{2}}{\partial t^{2}}\left(\frac{R_{\omega}}{r}\right)+\frac{\partial}{\partial r}\left(r^{2} f(r) \frac{\partial}{\partial r}\left(\frac{R_{\omega}}{r}\right)\right)-\left[\Omega r^{2}+l(l+1)\right] \frac{R_{\omega}}{r}=0
$$

where $\Omega$ is the eigenvalue of function $L(y)$. The fifth dimensional equation about $L(y)$ is

$$
\frac{d^{2} L(y)}{d y^{2}}+\Lambda \sqrt{\frac{\Lambda}{3}} \frac{d L(y)}{d y}+\Omega L(y)=0,
$$

which is discussed carefully in [16]. In the Randall-Sundrum double branes system, the modes along the extra dimension are quantized by means of stable standing waves, and then the eigenvalue is naturally discretized. The discrete spectra of $L(y)$ is

$$
L_{n}(y)=C e^{-\frac{\sqrt{3 \Lambda}}{2} y} \cos \left(n \pi \frac{y}{y_{1}}\right)
$$




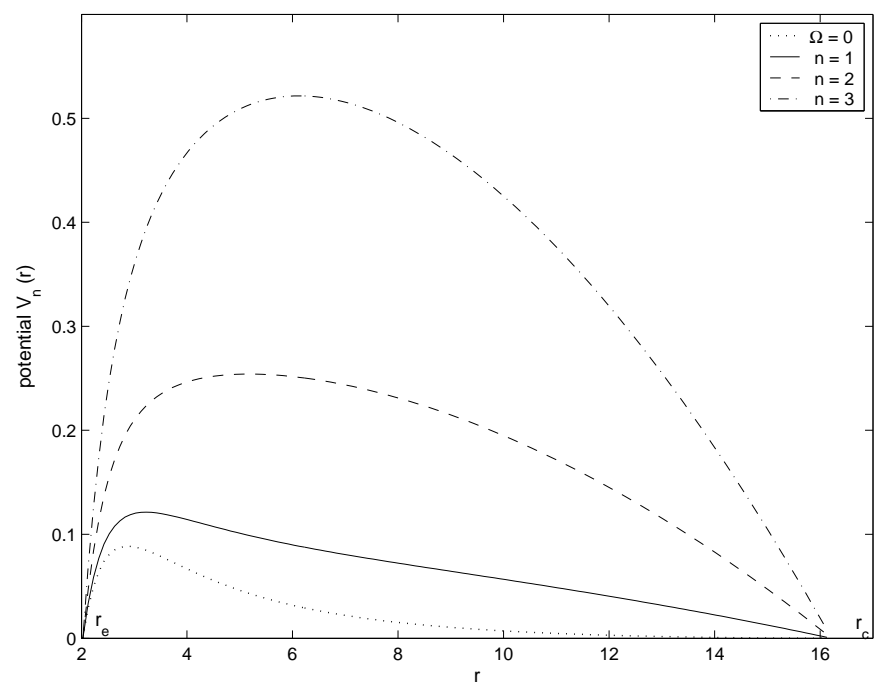

FIG. 1: The potentials $V_{n}(r)$ versus radial coordinate $\mathrm{r}$ with $\mathrm{n}=1$ (solid), $\mathrm{n}=2$ (dashed) and $\mathrm{n}=3$ (dash-dot). Meanwhile, we also draw the case of $4 \mathrm{D}(\Omega=0)$ with the dotted line for comparison. Here, we adopt $M=1, l=1, \Lambda=0.01$ and $y_{1}=10$ (a very large extra dimension).

and the quantum parameter $\Omega_{n}$ is

$$
\Omega_{n}=\frac{n^{2} \pi^{2}}{y_{1}^{2}}+\frac{3}{4} \Lambda,
$$

where $n=1,2,3 \cdots$ and $y_{1}$ is the thickness of the bulk.

\section{THE MASTER EQUATION FOR PROPAGATION OF SCALAR FIELD IN THE BULK}

It is known that radial direction determines the evolution of black hole radiation. The time variable of Eq. (7) can be removed by the Fourier component $e^{-i \omega t}$ via

$$
R_{\omega}(r, t) \rightarrow \Psi_{\omega l n}(r) e^{-i \omega t}
$$

where the subscript $n$ presents a new wave function unlike the usual $4 \mathrm{D}$ case $\Psi_{\omega l}$ [33]. Eq. (7) can be rewritten as

$$
\left[-f(r) \frac{d}{d r}\left(f(r) \frac{d}{d r}\right)+V(r)\right] \Psi_{\omega l n}(r)=\omega^{2} \Psi_{\omega l n}(r),
$$

whose potential function is given by

$$
V(r)=f(r)\left[\frac{1}{r} \frac{d f(r)}{d r}+\frac{l(l+1)}{r^{2}}+\Omega\right] .
$$

Now we introduce the tortoise coordinate

$$
x=\int \frac{d r}{f(r)} .
$$

The tortoise coordinate can be expressed with the surface gravity as follows

$$
x=\frac{1}{2 M}\left[\frac{1}{2 K_{e}} \ln \left(\frac{r}{r_{e}}-1\right)-\frac{1}{2 K_{c}} \ln \left(1-\frac{r}{r_{c}}\right)+\frac{1}{2 k_{o}} \ln \left(1-\frac{r}{r_{o}}\right)\right],
$$




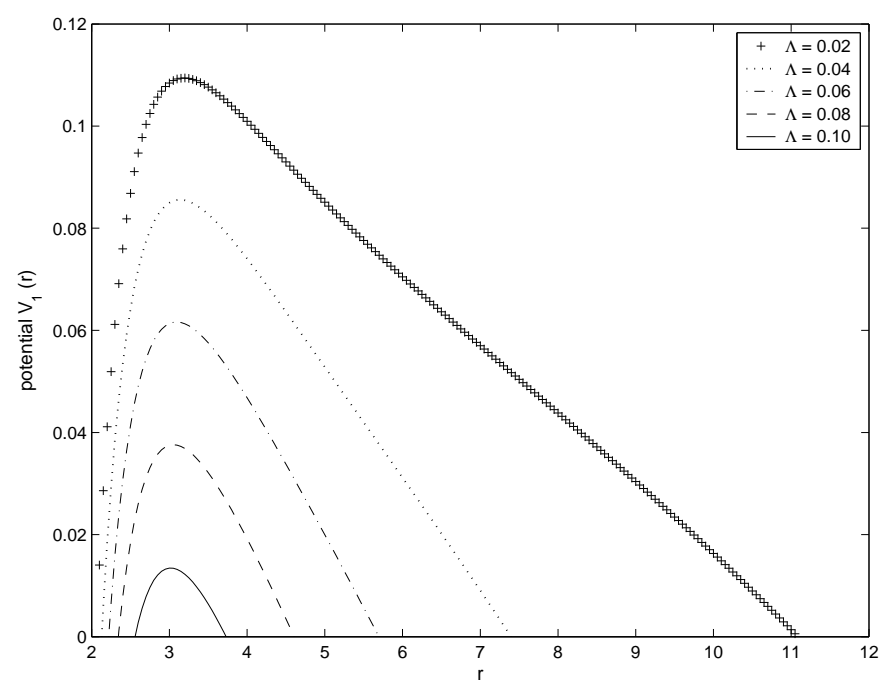

FIG. 2: The potentials $V_{1}(r)$ versus radial coordinate $\mathrm{r}$ with $\Lambda=0.02$ (plus), $\Lambda=0.04$ (dotted), $\Lambda=0.06$ (dash-dot), $\Lambda=0.08$ (dashed) and $\Lambda=0.10$ (solid). Here, we adopt $M=1, n=1, l=1$ and $y_{1}=10$ (a very large extra dimension).

where

$$
K_{i}=\frac{1}{2}\left|\frac{d f}{d r}\right|_{r=r_{i}}
$$

So under the tortoise coordinate transformation (14), the radial perturbation equation is obtained as

$$
\left[-\frac{d^{2}}{d x^{2}}+V(r)\right] \Psi_{\omega l n}(x)=\omega^{2} \Psi_{\omega l n}(x) .
$$

It is evident that Eq. (17) is exactly the same as Ragge-Wheeler equation in QNMs. The incoming or outgoing particle flowing between inner horizon $r_{e}$ and outer horizon $r_{c}$ is reflected and transmitted by the potential $V(r)$. Substituting the quantum parameters $\Omega_{n}$ (10) into Eq. (13), the quantum potentials are obtained as follows

$$
V_{n}(r)=f(r)\left[\frac{1}{r} \frac{d f(r)}{d r}+\frac{l(l+1)}{r^{2}}+\frac{n^{2} \pi^{2}}{y_{1}^{2}}+\frac{3}{4} \Lambda\right],
$$

which are illustrated in Fig. 1 and Fig. 2, The 5D potential contains the quantum number $n$ which is higher and thicker than the $4 \mathrm{D}$ 's when $\Omega=0$. Also, the height and the thickness of the former increase with bigger $n$. Meanwhile, for increasing cosmological constant $\Lambda$, the potential also becomes higher and thicker, and the interval between $r_{e}$ and $r_{c}$ is larger, too.

According to the quantum potential (18), the QNMs for massless scalar particles propagating in the black string space satisfy the boundary conditions [5] [6]

$$
\Psi_{\omega l n}(x) \approx C_{ \pm} \exp ( \pm i \omega x) \quad \text { as } x \longrightarrow \pm \infty
$$

denoting pure ingoing waves at the event horizon $r_{e}$ and pure outgoing waves at cosmological horizon $r_{c}$. 


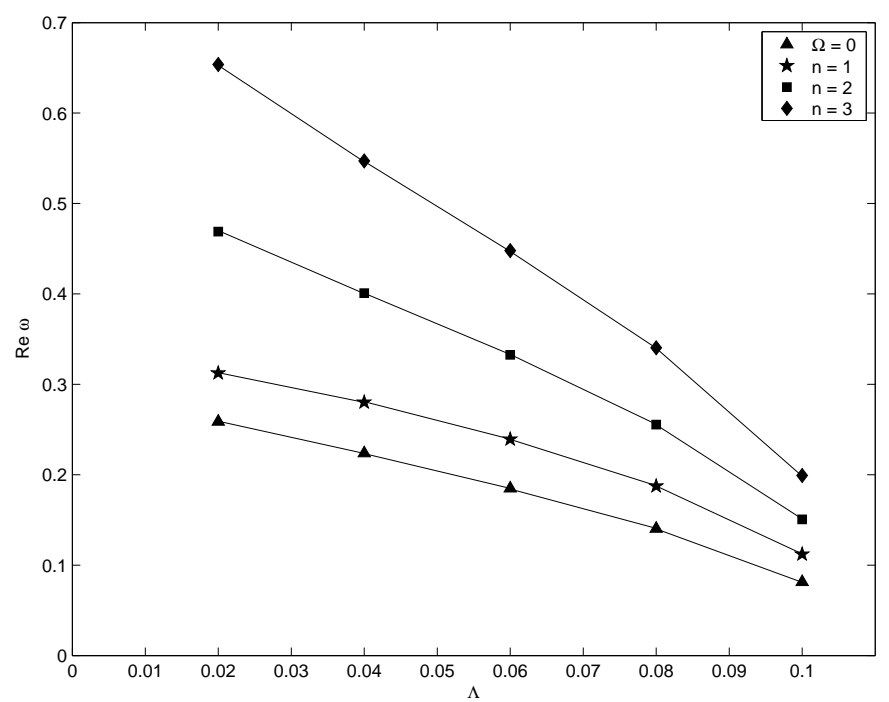

FIG. 3: The real parts of QN frequencies (Re $\omega$ ) of quasi-normal of the scalar field in 5D Ricci-flat black string space with $l=1, p=0, M=1$ and $y_{1}=10$. We denote pentagrams with $n=1$, squares with $n=2$, diamonds with $n=3$ and triangles with $\Omega=0$.

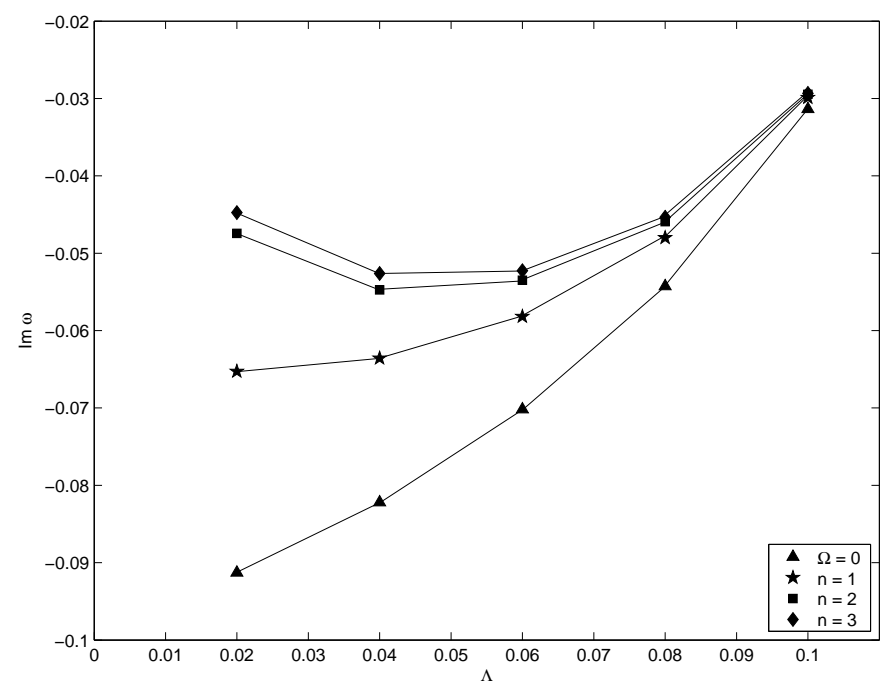

FIG. 4: The imaginary parts of QN frequencies ( $\operatorname{Im} \omega$ ) of quasi-normal of the scalar field in 5D Ricci-flat black string space with $l=1, p=0, M=1$ and $y_{1}=10$. We denote pentagrams with $n=1$, squares with $n=2$, diamonds with $n=3$ and triangles with $\Omega=0$.

\section{THE QN FREQUENCY OF MASSLESS SCALAR FIELD WITH THE THIRD ORDER WKB METHOD}

Numerical WKB approximation is an effective method to obtain the complex QN frequencies by using the wellknown Bohr-Sommerfeld rule. It was originally shown by Schutz et al 34] and was later developed to the third order by Iyer et al [35] 36] and to the sixth order by Konoplya [37]. Then after that this method is extensively used in 
various spaces [38]. The third order WKB formula for QN frequencies has the form [35] [36],

$$
\begin{gathered}
\omega^{2}=\left[V_{0}+\left(-2 V_{0}^{\prime \prime}\right)^{1 / 2} \tilde{\Lambda}\right]-i\left(p+\frac{1}{2}\right)\left(-2 V_{0}^{\prime \prime}\right)^{1 / 2}(1+\tilde{\Omega}), \\
p=\left\{\begin{array}{c}
0,1,2, \ldots, \operatorname{Re} \omega>0, \\
-1,-2,-3, \ldots, \operatorname{Re} \omega<0,
\end{array}\right.
\end{gathered}
$$

where

$$
\begin{aligned}
\tilde{\Lambda}= & \frac{1}{\left(-2 V_{0}^{\prime \prime}\right)^{1 / 2}}\left[\frac{1}{8}\left[\frac{V_{0}^{(4)}}{V_{0}^{\prime \prime}}\right]\left[\frac{1}{4}+\alpha^{2}\right]-\frac{1}{288}\left[\frac{V_{0}^{\prime \prime \prime}}{V_{0}^{\prime \prime}}\right]^{2}\left(7+60 \alpha^{2}\right)\right] \\
\tilde{\Omega}= & \frac{1}{\left(-2 V_{0}^{\prime \prime}\right)}\left[\frac{5}{6912}\left[\frac{V_{0}^{\prime \prime \prime}}{V_{0}^{\prime \prime}}\right]^{4}\left(77+188 \alpha^{2}\right)-\frac{1}{384}\left[\frac{V_{0}^{\prime \prime \prime} V_{0}^{(4)}}{V_{0}^{\prime \prime} 3}\right]\left(51+100 \alpha^{2}\right)+\frac{1}{2304}\left[\frac{V_{0}^{(4)}}{V_{0}^{\prime \prime}}\right]^{2}\left(67+68 \alpha^{2}\right)\right. \\
& \left.+\frac{1}{288}\left[\frac{V_{0}^{\prime \prime \prime} V_{0}^{(5)}}{V_{0}^{\prime \prime 2}}\right]\left(19+28 \alpha^{2}\right)-\frac{1}{288}\left[\frac{V_{0}^{(6)}}{V_{0}^{\prime \prime}}\right]\left(5+4 \alpha^{2}\right)\right]
\end{aligned}
$$

where $\alpha=\mathrm{p}+1 / 2$ and symbol $p$ is the various overtones. The primes and superscript $(n)$ denote differentiation with respect to the tortoise coordinate $x$. The subscript 0 on a variable denotes the value at $x_{0}$, which is the position of maximum $V(x)$, namely, $V_{0}^{(n)}=\left.\frac{d^{n} V}{d x^{n}}\right|_{x=x_{0}}$. Substituting potential (18) into $\tilde{\Lambda}$ and $\tilde{\Omega}$, we can obtain the vital QN frequencies for the massless scalar field in the 5D black string space. Meanwhile, it is known that the WKB approximation is accurate for the low-lying QNM modes, but it fails to calculate the higher-order modes. Therefore, the condition $l>p$ is employed and the QN frequencies of fundamental key cases: $(l=1, p=0),(l=2, p=0)$ and $(l=2, p=1)$ are listed in the Table I, II and III, respectively. Meanwhile, potential (13) illustrate clearly that when $\Omega=0$ the Regge-Wheeler equation (17) is naturally reduced to 4D SdS case. So those tables also include a comparison with the results of $4 \mathrm{D}$ case. Here we should notice that the denotation $\Omega=0$ does not indicates $n=0$. To avoid the confusion about parameters $\Omega$ and $\Omega_{n}$, we provide some explanation in the conclusion part. Here, we adopt $M=1$ and $y_{1}=10$ and analyse the QN frequencies from two aspects: cosmological constant $\Lambda$ and quantum number $n$.

TABLE I: The QN frequencies of massless scalar field for $l=1$ and $p=0$

\begin{tabular}{|c|c|c|c|c|}
\hline \hline$\Lambda$ & $\omega(n=1)$ & $\omega(n=2)$ & $\omega(n=3)$ & $\omega(\Omega=0)$ \\
\hline 0.02 & $0.312415-0.0652835 i$ & $0.468963-0.0474421 i$ & $0.653750-0.0447420 i$ & $0.258832-0.091277 i$ \\
\hline 0.04 & $0.280096-0.0635772 i$ & $0.400800-0.0547428 i$ & $0.547038-0.0526103 i$ & $0.223730-0.082191 i$ \\
\hline 0.06 & $0.239281-0.0581832 i$ & $0.332630-0.0534970 i$ & $0.447846-0.0522556 i$ & $0.184973-0.070180 i$ \\
\hline 0.08 & $0.187333-0.0479931 i$ & $0.255402-0.0459195 i$ & $0.340367-0.0453565 i$ & $0.140302-0.054267 i$ \\
\hline 0.10 & $0.112055-0.0298885 i$ & $0.150672-0.0294599 i$ & $0.199165-0.0293426 i$ & $0.081436-0.031372 i$ \\
\hline \hline
\end{tabular}

Firstly, for a given cosmological constant $\Lambda$, it is shown that the real parts of QNMs (Re $\omega)$ increase with bigger quantum number $n$. But the absolute value of the imaginary parts $(|\operatorname{Im} \omega|)$ decrease for bigger $n$. In general, the actual frequencies in 5D are larger than 4D's, and the scalar field in 5D decays more slowly than the one in 4D. With increasing $n$, the QN frequencies become larger and the scalar field decays more slowly.

Secondly, for a given $n$ we can also read that $\operatorname{Re} \omega$ and $|\operatorname{Im} \omega|$ decrease with larger $\Lambda$. It means that with the increasing cosmological constant $\Lambda$ the actual frequency becomes smaller and the scalar field decays more slowly. 


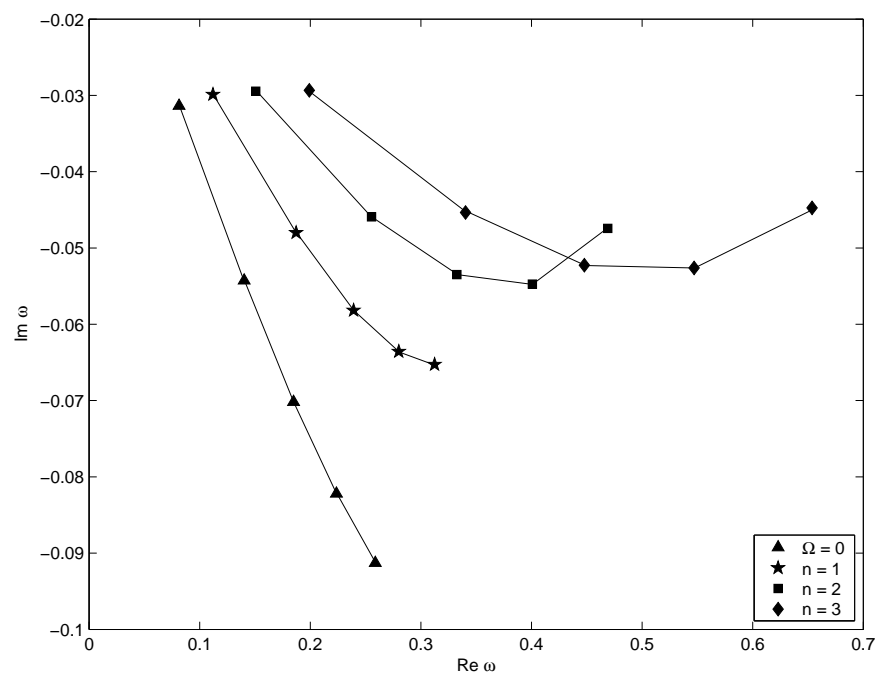

FIG. 5: The imaginary parts $(\operatorname{Im} \omega)$ versus the real parts $(\operatorname{Re} \omega)$ in $5 \mathrm{D}$ Ricci-flat black string space with $l=1, p=0, M=1$ and $y_{1}=10$. We denote pentagrams with $n=1$, squares with $n=2$, diamonds with $n=3$ and triangles with $\Omega=0$.

TABLE II: The QN frequencies of massless scalar field for $l=2$ and $p=0$

\begin{tabular}{|c|c|c|c|c|}
\hline \hline$\Lambda$ & $\omega(n=1)$ & $\omega(n=2)$ & $\omega(n=3)$ & $\omega(\Omega=0)$ \\
\hline 0.02 & $0.468382-0.0791272 i$ & $0.561831-0.0581448 i$ & $0.715167-0.0470975 i$ & $0.434260-0.0886175 i$ \\
\hline 0.04 & $0.415397-0.0714952 i$ & $0.494992-0.0605678 i$ & $0.614782-0.0546797 i$ & $0.380534-0.0787860 i$ \\
\hline 0.06 & $0.353220-0.0620269 i$ & $0.417977-0.0565769 i$ & $0.512001-0.0535286 i$ & $0.319866-0.0668696 i$ \\
\hline 0.08 & $0.276190-0.0494536 i$ & $0.324789-0.0472241 i$ & $0.394093-0.0459458 i$ & $0.247382-0.0519274 i$ \\
\hline 0.10 & $0.165316-0.0301362 i$ & $0.193354-0.0297108 i$ & $0.232939-0.0294638 i$ & $0.146577-0.0306956 i$ \\
\hline \hline
\end{tabular}

TABLE III: The QN frequencies of massless scalar field for $l=2$ and $p=1$

\begin{tabular}{|c|c|c|c|c|}
\hline \hline$\Lambda$ & $\omega(n=1)$ & $\omega(n=2)$ & $\omega(n=3)$ & $\omega(\Omega=0)$ \\
\hline 0.02 & $0.443126-0.242565 i$ & $0.527013-0.162412 i$ & $0.718255-0.141176 i$ & $0.420199-0.268921 i$ \\
\hline 0.04 & $0.400020-0.215866 i$ & $0.482499-0.177642 i$ & $0.615655-0.163493 i$ & $0.371026-0.238251 i$ \\
\hline 0.06 & $0.345271-0.186311 i$ & $0.413660-0.168533 i$ & $0.512501-0.160352 i$ & $0.313821-0.201780 i$ \\
\hline 0.08 & $0.273104-0.148377 i$ & $0.323641-0.141410 i$ & $0.394371-0.137765 i$ & $0.244094-0.156371 i$ \\
\hline 0.10 & $0.164801-0.0904138 i$ & $0.193234-0.0891112 i$ & $0.233009-0.0883807 i$ & $0.145667-0.092201 i$ \\
\hline \hline
\end{tabular}

These results are in agreement with the results of 4D SdS case with the sixth order WKB method 39]. As mentioned above, the two circumstances for given $n$ and $\Lambda$ can be manifested in Fig. 3 and Fig. 4 which are obtained by Table I.

In order to study the relationship between the actual frequency and the damping of the oscillation, we also plot the Im $\omega$ versus Re $\omega$ graph in Fig. [5. Obviously, the absolute value of the imaginary parts increase entirely with the larger real parts. However, when the quantum number $n$ becomes larger, there is a break point in the curve. In other words, $|\operatorname{Im} \omega|$ does not monotonously increase with $\operatorname{Re} \omega$ for bigger $n$, especially in the case of $n=2,3$.

To explain the reason, we should refer to this black string's reflection (or transmission) [17]. In the square barrier 
model [17], the reflection or transmission coefficients have the analytic forms. As one knows that the reflection should be stronger with higher barriers. The reflection coefficients of $n=2,3$ would be larger than the cases of $n=1$ or $\Omega=0$ in the usual viewpoint. On the contrary, the values of reflection coefficients of $n=2,3$ are smaller than the cases of $n=1$ or $\Omega=0$. If the resonant effect of quantum mechanics exists in the barriers, the peculiar behavior is easily to be gotten. Mathematically, an oscillatory cosine function $\cos (2 k 2 d)$ is contained in the expression of reflection coefficients $R$ or transmission coefficients $T$ [17]. (For detailed discussion of those behavior, see Ref. [17].) The scatting potential of $n=2,3$ also have the peculiar QN frequencies by the same resonant effect. From Fig. 1, we can read that with larger $n$ the peak of potential slips the cosmological horizon and the potential becomes higher and wider, especially in the case of $n=2,3$. Hence with the enhancing of resonant effect the QN frequencies decay more slowly, even though the QN frequencies become larger. So the anomalous values of $n=2,3$ are the result of resonant effect of quantum mechanics in the barriers.

\section{CONCLUSION}

In this paper, we have used the third-order WKB approximation to calculate the quasi-normal frequencies of massless scalar field outside a 5D black string. We summarize what has been achieved.

1. In this 5D Ricci-flat black string space, the QNMs is studied by fixing either the cosmological constant $\Lambda$ or the quantum number $n$. From the result we find that the scalar field decays more slowly with the increasing $\Lambda$ or $n$. For a given cosmological constant $\Lambda$, the $5 \mathrm{D}$ actual frequency becomes bigger with increasing $n$. While for a given $n$, the frequency becomes smaller with bigger cosmological constant $\Lambda$. In other words, the $5 \mathrm{D}$ QN frequencies are larger than 4 D's $(\Omega=0)$.

2. As one candidate of the higher dimensional black hole, the 5D Ricci-flat black string implies us something interesting. The quantum number $n$ depicts a new wave solution $\Psi_{\omega l n}$ of Schrödinger wavelike equation. The spectrum of original potential is discrete for the existence of quantum number $n$. The non-trivial radiation can reveal much of valuable information that characterizes the higher dimensional background, such as the dimensionality of space, the topological structure and so on. Here, the QN frequencies of 5D Ricci-flat black string are determined by the black hole mass $M$, the effective cosmological constant $\Lambda$, the quantum number $n$ and the thickness of the bulk $y_{1}$. The information about extra dimension is encoded in those QN frequencies such as the magnitude of extra dimension and the thickness of the bulk. It is known that the best method to probe black hole is the detectable QN spectrum. If extra dimension does exist and is visible near black hole, maybe those QN frequency can prove its existence.

3. To ensure the validity of results, tables and figures, we use Mathematica software to design a program and calculate carefully those QN frequencies. The induced four dimensional results $(\Omega=0)$ are exactly identical with 4D SdS black hole's [39]. Otherwise, just as 4D case [39], the QN frequencies decrease with increasing cosmological constant $\Lambda$. Of course, this program is tested in some other black holes such as 4D Schwarzschild black hole [36], and the same results are got which is not presented in this paper. This method gets desired effects and hence believable. .

4. The reason why we discuss, not the gravitational perturbation but a test scalar field, is that this paper is a continuation of previous work [16] to a certain extent. As we known from the spirit of string theory, the standard model fields are confined on 3-brane except for gravitons and scalar particles. The original goal to introduce the scalar field is to examine the effect of an extra dimension on black hole radiation. Surprisingly, the radial component 
of 5D Klein-Gordon equation can be rewritten exactly as the Regge-Wheeler form. Considering the QNMs boundary condition, we have studied the spectrum of QN frequencies by usual third order WKB method. After all, the QNMs of higher dimensional black hole is very attractive and interesting. Certainly, the basic gravitation field is easier to be detected by gravitational wave than other fields. Anyway, it is interesting to study this case and further work is needed.

5. It should be noticed that the parameter $\Omega$ is not the same as $\Omega_{n}$. In fact, parameter $\Omega$ is introduced to separate the variables $R_{\omega}(r, t), L(y)$ and $Y_{l m}(\theta, \phi)$ in this paper. But parameter $\Omega_{n}$ is a particular eigenvalue which is deduced from the original $\Omega$ under the standing wave condition [16],

$$
y_{1} \sqrt{\Omega-\frac{3}{4} \Lambda}=n \pi
$$

In other words, $\Omega$ is a free parameter, while $\Omega_{n}=\frac{n^{2} \pi^{2}}{y_{1}^{2}}+\frac{3}{4} \Lambda$ is constrained by $n, y_{1}$ and $\Lambda$. Meanwhile, the quantum number $n$ is a positive integer i.e. $n>0$ according to the condition (24) 16]. Furthermore, cosmological constant $\Lambda$ is nonzero in the de Sitter universe. So considering the conditions $n>0$ and $\Lambda>0$, we can get the eigenvalue $\Omega_{n}>0$. By the way, when $n=0$ the steady stand wave (9) can not be formed and this case must be abandoned. On the other hand, the potential function (13) indicates that the case $\Omega=0$ (not $\mathrm{n}=0$ ) corresponds to usual 4D SdS. Hence, the notations $\Omega=0$ in figures and tables just show the one of $4 \mathrm{D}$ SdS. Of course, some other notation could be used in principle.

\section{Acknowledgments}

Project supported by the National Basic Research Program of China (2003CB716300), National Natural Science Foundation of China (10573003) and National Natural Science Foundation of China (10573004). We are grateful to Feng Luo for useful help.

[1] T. Regge and J. Wheeler, Phys. Rev. 108 (1957) 1063.

[2] Vishveshwara, C.V., Nature, 227 (1970) 936.

[3] Press, W.H., Astrophys. J., 170 (1971) L105.

[4] Chandrasekhar, S., Proc. R. Soc. London, Ser. A, 343 (1975) 289; H. -J. Blome and B. Mashhoon, Phys. Lett. 100A (1984) 231; V. Ferrari and B. Mashhoon, Phys. Rev. D30 (1984) 295; E. W. Leaver, Proc. R. Soc. London, Ser. A, 402 (1985) 285; H. -P. Nollert, and B. G. Schmidt, Phys. Rev. D45 (1992) 2617; H. Y. Liu and B. Mashhoon, Class. Quant. Grav. 13 (1996) 233; V. Cardoso and J. P. S. Lemos, Phys. Rev. D64 (2001) 084017.

[5] H. P. Nollert, Class. Quant. Grav. 16 (1999) R159.

[6] K. D. Kokkotas and B. G. Schmidt, Living Reviews Relativ. 2 (1999) 2 , gr-qc/9909058v1.

[7] P. S. Wesson, Space-Time-Matter (1999) (World Scientific Publishing Co. Pte. Ltd, Singapore).

[8] J. M. Overduin and P. S. Wesson, Phys. Rep. 283 (1997) 303, gr-qc/9805018.

[9] A. Macias, G. J. Fuentes y Martinez and O. Obregon, Gen. Rel. Grav. 25 (1993) 549.

[10] P. H. Lim and P. S. Wesson, Astrophys. J. 397 (1992) L91.

[11] A. Billyard, P. S. Wesson and D. Kalligas, Int. J. Mod. Phys. D4 (1995) 639.

[12] H. Y. Liu and P. S. Wesson, Phys. Lett. B381 (1996) 420. 
[13] H. Y. Liu, Gen. Rel. Grav. 23 (1991) 759.

[14] H. Y. Liu and P. S. Wesson, J. Math. Phys. 33, 3888 (1992).

[15] B. Mashhoon, H. Y. Liu and P. S. Wesson, Phys. Lett. B331 (1994) 305.

[16] M. L. Liu, H. Y. Liu, L. X. Xu and P. S. Wesson, Mod. Phys. Lett. A21 (2006) 2937, gr-qc/0611137.

[17] M. L. Liu, H. Y. Liu, F. Luo and L. X. Xu, Gen. Rel. Grav. 39 (2007) 1389, gr-qc/0705.2465.

[18] H. Y. Liu, and P. S. Wesson, Astrophys. J. 538 (2000) 386, gr-qc/0003034.

[19] N. Arkani-Hamed, S. Dimopoulos, and G. R. Dvali, Phys. Lett. B429 (1998) 263, hep-ph/9803315; Phys. Rev. D59 (1999) 086004, hep-ph/9807344 I. Antoniadis, N. Arkani-Hamed, S. Dimopoulos, and G. R. Dvali, Phys. Lett. B436 (1998) 257, hep-ph/9803315.

[20] L. Randall and R. Sundrum, Phys. Rev. Lett. 83 (1999) 4690, hep-th/9906064

[21] L. Randall and R. Sundrum, Phys. Rev. Lett. 83 (1999) 3370, hep-ph/9905221

[22] A. Chamblin, S. W. Hawking, and H. S. Reall, Phys. Rev.D 61, 065007 (2000); N. Dadhich, R. Maartens, P. Papadopoulos and V. Rezania, Phys. Lett. B487 (2000) 1, hep-th/0003061v3.

[23] de Leon J. Ponce, Mod. Phys. Lett. A16 (2001) 2291, gr-qc/0111011.

[24] S. S. Seahra, Phys. Rev. D68 (2003) 104027, hep-th/0309081

[25] H. Y. Liu, Phys. Lett. B560 (2003) 149, hep-th/0206198.

[26] R. G. Emparan, T. Horowitz, and R. C. Myers, Phys. Rev. Lett. 85 (2000) 499, hep-th/0003118, S. Dimopoulos and G. Landsberg, Phys. Rev. Lett. 87 (2001) 161602, hep-ph/0106295, S. B. Giddings and S. Thomas, Phys. Rev. D65 (2002) 056010, hep-ph/0106219.

[27] V. Cardoso, J. P. S. Lemos, and S. Yoshida, JHEP 0312 (2003) 041, hep-th/0311260 V. Cardoso, J. P. S. Lemos, and S. Yoshida, Phys. Rev. D69 (2004) 044004; P. Kanti and R. A. Konoplya, Phys. Rev. D73 (2006) 044002, hep-th/0512257 R. A. Konoplya, Phy. Rev. D 68 (2003) 124017, hep-th/0309330 P. Kanti, R. A. Konoplya and A. Zhidenko, Phys. Rev. D74 (2006) 064008, gr-qc/0607048

[28] Mashhoon, B., Wesson, P. S. and Liu, H. Y., Gen. Rel. Grav. 30 (1998) 555.

[29] Wesson, P. S., Mashhoon. B., Liu, H. Y. and Sajko, W. N., Phys. Lett. B456 (1999) 34.

[30] H. Y. Liu and B. Mashhoon, Phys. Lett. A272 (2000) 26, gr-qc/0005079.

[31] B. Mashhoon and P. S. Wesson, Class. Quant. Grav. 21 (2004) 3611, gr-qc/0401002.

[32] Y. L. Ping, H. Y. Liu and L. X. Xu, Int. J. Mod. Phys. A22 (2007) 985, gr-qc/0610094.

[33] I. Brevik and B. Simonsen, Gen. Rel. Grav. 33 (2001) 1839.

[34] B. F. schutz and C. M. Will, Astrophys. J. Letter. Ed. 291 (1985) L33.

[35] S. Iyer and C. M. Will, Phys. Rev. D35 (1987) 3621.

[36] S. Iyer, Phys. Rev. D35 (1987) 3632.

[37] R. A. Konoplya, Phys. Rev. D68 (2003) 024018 gr-qc/0303052.

[38] R. A. Konoplya, Gen. Rel. Grav. 34 (2002) 329, gr-qc/0109096, O. B. Zaslavskii, Phys. Rev. D43 (1991) 605; L. E. Simone and C. M. Will, Class. Quant. Grav. 9 (1992) 963; K. Kokkotas, and B. F. Schutz, Phys. Rev. D37 (1988) $3378 ;$ E. Berti and K. Kokkotas, Phys. Rev. D67 (2003) 064020, gr-qc/0301052

[39] A. Zhidenko, Class. Quant. Grav. 21 (2004) 273, gr-qc/0307012. 